\title{
Potency and Antibacterial Efficacy of Degraded Chloramphenicol in Marketed Eye Drop Preparations
}

\author{
Md. Shamim Ahmed ${ }^{1}$, Sultana Rajia ${ }^{2}$, Md. Ajijur Rahman ${ }^{1}$ and Md. Anwar Ul Islam ${ }^{1}$ \\ ${ }^{1}$ Department of Pharmacy, University of Rajshahi, Rajshahi-6205, Bangladesh \\ ${ }^{2}$ Molecular Biology Laboratory, Institute of Biological Sciences, University of Rajshahi, Rajshahi-6205, Bangladesh
}

\begin{abstract}
This study was done to evaluate the antibacterial activity of chloramphenicol eye drops after exposure to sunlight as well as to measure the percentage of drug content. Chloramphenicol eye drops manufactured by four different companies were collected from local market and exposed to sunlight and their antimicrobial activity was determined against Salmonella typhi and Staphylococcus aureus. Each product was exposed to sunlight for fourteen hours in three different ways with or without of their packaging. The percentage of chloramphenicol contents in eye drop samples was also measured by UV-spectrophotometric method. Significant differences in the inhibitory zones were observed among different set of samples. An inverse correlation between percentage of chloramphenicol content and their antibacterial activity was observed. The results indicate that the UVspectrophotometric method cannot distinguish between chloramphenicol and its degraded products and thus gives incorrect high values of drug content in the preparations, therefore, it should not be considered as an appropriate method to analyze chloramphenicol preparation with its degradation products.
\end{abstract}

Keywords: Chloramphenicol eye drops, sunlight-induced degradation, antibacterial activity.

\section{Introduction}

Chloramphenicol (MF: $\mathrm{C}_{11} \mathrm{H}_{12} \mathrm{C}_{12} \mathrm{~N}_{2} \mathrm{O}_{5}$, MW: 323.1) is a broad spectrum antibiotic which is effective against a wide range of gram-negative and gram-positive organisms (Lawrence, 1987). It is still widely used as eye drops for the treatment of bacterial conjunctivitis caused by Escherichia coli, Haemophilus influenzae, Staphylococcus aureus, Streptococcus haemolyticus, Morax-Axenfield and others. Chloramphenicol is also indicated in typhoid fever, various salmonella infections, anaerobic infections with Bacteroides fragilis, rickettsial infections, and bacterial meningitis. It has been the drug of choice for the treatment of $H$. injluenzae meningitis, especially if the organism is a B-Lactarnase producer (Turk, 1977, Martindale, 1989).

Chloramphenicol is highly sensitive to light and photochemical decomposition of chloramphenicol results in a yellowing of the solution, development of an orange-yellow precipitate and lowering of the $\mathrm{pH}$ (Martindale, 1989). The chemistry of the photo-degradation products of chloramphenicol aqueous solution at room temperature $\left(21-30^{\circ} \mathrm{C}\right)$ and in the presence of sunlight, suggest that under the influence of light and water the drug undergoes oxidation, reduction and condensation reactions. The photochemical degradation of chloramphenicol in various solvents was investigated and the following photolysis products isolated: hydrochloric acid, 4-nitrobenzaldehyde, 4nitrobenzoic acid, 4,4-azoxybenzoic acid and 2-amino-1-(4nitrophenyl) propane-1,3-diol, as indicated by thin layer chromatography (Shih, 1971). Later work concerning the quality control of a pharmaceutical preparation ( $0.24 \%$ eye drops), it has found that under the influence of sunlight, some nitrosocompounds: p-nitrobenzene, p-nitrobenzaldehyde and pnitrosobenzoic acid are formed.

In this article we show for the first time that antimicrobial activity was decreased in the sunlight -induced degradation of chloramphenicol preparations whereas the concentrations of chloramphenicol were increased significantly compared to control when the content was analyzed by UVspectrophotometric method. The results of this study suggest that UV-spectrophotometric method may lead to incorrect results as the degraded products of chloramphenicol also absorb UV radiation around the same wavelength.

\footnotetext{
*Correspondence to: Md. Anwar UI Islam, Department of Pharmacy, University of Rajshahi, Rajshahi-6205, Bangladesh. Tel: 880721-711110, E-mail: profanwarulislam@yahoo.com
} 


\section{Materials and Methods}

Materials: The chloramphenicol $0.5 \%$ eye drops of four different companies (A, B, C and D) were collected from local market of Rajshahi City. Sterilized filter paper discs $(6 \mathrm{~mm})$ and commercially available nutrient agar medium were purchased from the local suppliers of DIFCO and BBL (U.S.A). All the reagents used were of analytical grades. A double beam spectrophotometer UV3600 (Shimadzu Corporation, Japan) was used to take Spectrophotometric readings.

The microorganisms were collected from the Institute of Nutrition and Food Science (INFS), University of Dhaka and International Center for Diarrheal Disease Research, Bangladesh (ICDDR, B).

Experimental design and preparation and treatment of samples with sunlight and heat: A $3 \mathrm{ml}$ aliqout of the four different samples (commercial preparations of $0.5 \%$ chloramphenicol eye drops, A, B, C and D) were used in this study. Each preparation was divided into four different sets and exposed to sunlight for 14 hours in divided days:

Set-1: Sample (3ml) stored in refrigerator with their containers (control).

Set-2: Sample ( $3 \mathrm{ml})$ exposed to sunlight with their primary and secondary packaging (container + carton).

Set-3: Sample (3ml) exposed to sunlight with their container only (primary packaging).

Set-4: Sample $(3 \mathrm{ml})$ exposed to sunlight in clear transparent glass test tubes (direct exposure).

Monitoring of change in color and determination of antimicrobial activity of sunlight-treated samples: The change in the appearance after sunlight-induced degradation of chloramphenicol preparations was noted before and after of exposure. The antibacterial activity of sunlight-treated chloramphenicol $(0.5 \%)$ eye drops were evaluated by the disc diffusion method against two bacteria - Staphylococcus aureus and Salmonella Typhi (Bauer, 1966) at $30 \mu \mathrm{g} /$ disc. For preparation of 30 $\mu \mathrm{g} / \mathrm{discs}$ of chloramphenicol preparations, $6 \mu \mathrm{l}$ of each solution were taken by a $10 \mu \mathrm{l}$ micropipette in an aseptic condition. The discs were placed on test organism seeded plates. The activity was determined after 24 hours of incubation at $37^{\circ} \mathrm{C}$. The diameter of the zone was measured with a transparent scale in $\mathrm{mm}$.
Analysis of chloramphenicol content in the samples by $U V$-Spectroscopic method: The potency of heat-induced degraded chloramphenicol aqueous solution was determined using UV-spectrophotometric method. $2 \mathrm{ml}$ solution (10 mg equivalent of chloramphenicol) from each preparation were taken in $100 \mathrm{ml}$ measuring cylinder. Then $50 \mathrm{ml}$ of distilled water were added and stirred for 5 minutes. The total volume was made up to $100 \mathrm{ml}$ with the same solvent and mixed well. $10 \mathrm{ml}$ solution was transferred in another $100 \mathrm{ml}$ measuring cylinder and the volume was adjusted with the same solvent. The absorbance of the assay preparation was taken at $278 \mathrm{~nm}$ wavelength in a UV-Spectrophotometer and the results are recorded and percentage of drug content was calculated.

\section{Results and Discussion}

Change in appearance and antibacterial activity after exposure to sun: Before exposure to sun the appearances of all the samples of different sets were clear and colorless. After 14 hours exposure of sunlight the color of all samples of Set-2 turned into opaque except the sample from D retained its original color. In case of Set-3, the color of all of the sample solutions turned into light yellow to deep yellow (Table 1). The results suggest that packaging is very important factor for the stability of chloramphenicol $0.5 \%$ eye drops. The color of the set- 2 samples did not change significantly because of protection of light by the secondary packaging (carton). On the other hand, the container alone is not enough to protect the sunlight which is evident by the production of colored degradation products in most of the samples in Set - 3 . The change in appearance of the samples in Set- 4 was most prominent and all of the preparations turned to deep yellow.

The zone of inhibition of Set - 2, Set - 3 and Set - 4 samples were compared to that of Set-1 after exposure to sunlight against Salmonella Typhi and Staphylococcus aureus and the results are shown in Table 1. It is evident that the antibacterial activity decreased from Set-1 to Set-4 (Figure-1 and Figure 2). The measured zone of inhibition of Set-1, Set-2, Set-3 and Set-4 of product B against $S$. aureus was 21.27, 20.19, 19.92 and 17.75, respectively. The percentage decrease of activity of Set- 4 with respect to Set-1 was $16.54 \%$ against $S$. aureus. We have observed the almost similar trend in other two products $\mathrm{C}$ and $\mathrm{D}$. In 
a previous study it was observed that inadequate refrigeration and prolonged shelf lives of chloramphenicol generics collected in India were associated with very high levels of chloramphenicol thermal breakdown product, 2amino-1-(4-nitrophenyl) propane-1,3-diol, when compared with drops stored in proper (Serrano, 2004).

Table 1. Estimated potency and antibacterial activity of chloramphenicol $(0.5 \%)$ eye drops after treatment with sunlight.

\begin{tabular}{|c|c|c|c|c|c|}
\hline \multirow{2}{*}{$\begin{array}{l}\text { Product } \\
\text { name }\end{array}$} & \multirow[t]{2}{*}{ Sets } & \multirow{2}{*}{$\begin{array}{l}\text { Change of appearance } \\
\text { after exposure to sunlight }\end{array}$} & \multirow{2}{*}{$\begin{array}{l}\text { Percentage drug } \\
\text { content }(\%)\end{array}$} & \multicolumn{2}{|c|}{ Zones of inhibition at $30 \mu \mathrm{g} / \mathrm{disc}(\mathrm{mm})$} \\
\hline & & & & S. typhi & S. aureus \\
\hline \multirow[t]{4}{*}{ A } & Set - 1 & - & $112.79 \pm 0.66$ & $11.83 \pm 0.59$ & $20.66 \pm 0.94$ \\
\hline & Set - 2 & Colorless, opaque & $118.52 \pm 1.7$ & $10.31 \pm 0.64$ & $19.54 \pm 0.56$ \\
\hline & Set - 3 & Yellowish & $122.22 \pm 0.91$ & $9.72 \pm 0.53$ & $19.43 \pm 1.06$ \\
\hline & Set -4 & Deep yellow & $139.05 \pm 1.97$ & $7.97 \pm 0.99$ & $18.11 \pm 0.95$ \\
\hline \multirow[t]{4}{*}{ B } & Set -1 & - & $108.42 \pm 0.63$ & $11.64 \pm 0.34$ & $21.27 \pm 0.23$ \\
\hline & Set - 2 & Colorless, opaque & $110.77 \pm 1.4$ & $10.40 \pm 0.9$ & $20.19 \pm 0.76$ \\
\hline & Set -3 & Yellowish & $112.50 \pm 2.03$ & $9.94 \pm 1.1$ & $19.92 \pm 0.8$ \\
\hline & Set -4 & Deep yellow & $140.40 \pm 1.9$ & $8.29 \pm 0.62$ & $17.75 \pm 0.48$ \\
\hline \multirow[t]{4}{*}{$\mathrm{C}$} & Set -1 & - & $107.74 \pm 0.56$ & $10.78 \pm 0.38$ & $22.04 \pm 0.05$ \\
\hline & Set - 2 & Colorless, opaque & $113.80 \pm 0.48$ & $9.68 \pm 0.29$ & $20.73 \pm 0.65$ \\
\hline & Set -3 & Yellow & $116.83 \pm 1.5$ & $9.24 \pm 0.7$ & $20.69 \pm 0.54$ \\
\hline & Set -4 & Deep yellow & $136.03 \pm 0.96$ & $7.36 \pm 1.1$ & $18.69 \pm 1.1$ \\
\hline \multirow[t]{4}{*}{ D } & Set -1 & - & $101.68 \pm 0.55$ & $13.12 \pm 0.83$ & $24.25 \pm 0.22$ \\
\hline & Set - 2 & Colorless, slightly opaque & $103.37 \pm 2.0$ & $11.60 \pm 0.53$ & $21.87 \pm 0.95$ \\
\hline & Set -3 & Yellow & $105.60 \pm 0.37$ & $11.39 \pm 0.62$ & $20.88 \pm 1.1$ \\
\hline & Set -4 & Deep yellow & $124.58 \pm 1.6$ & $9.42 \pm 0.55$ & $18.52 \pm 0.5$ \\
\hline
\end{tabular}

Values are expressed as mean $\pm \mathrm{SD}$ of three replicate

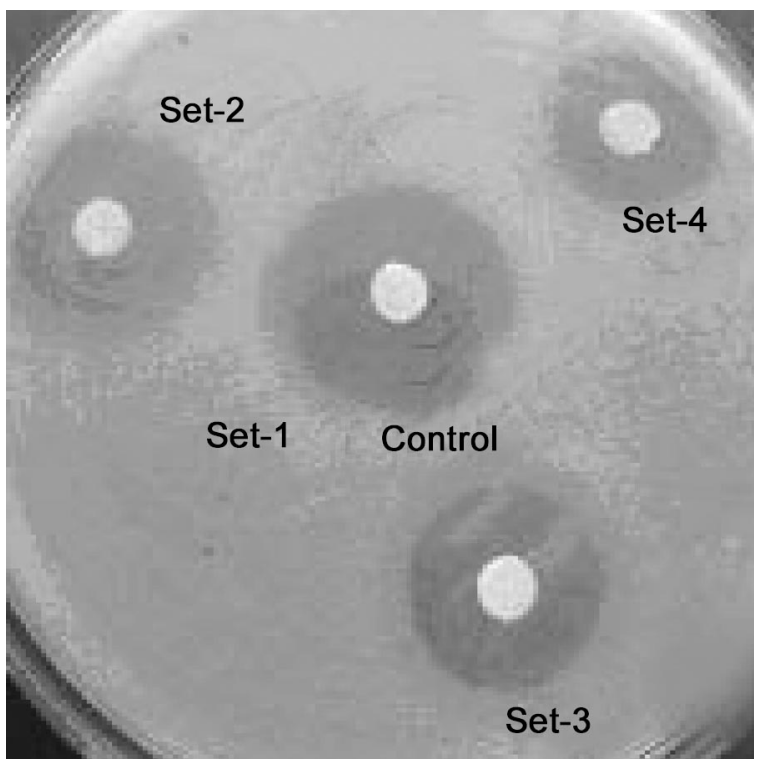

Figure 1. Antibacterial activity of different sets of samples of product B against Staphylococcus aureus at $30 \mu \mathrm{g} / \mathrm{disc}$. Where, Set-1: sample stored in refrigerator with their containers (control); Set-2: sample exposed to sunlight with their container and carton, Set-3: sample exposed to sunlight with their container only; Set-4: sample exposed to sunlight in clear transparent glass test tubes (direct exposure).

Change in percentage of drug content after exposure to sun: In all cases the measured percentage of drug content in the control samples were more than $100 \%$. When the samples were treated with sunlight for around 14 hours, the percentage drug content was increased. For example, the $\%$ content of chloramphenicol in the Set-1 of the product B was $108.42 \%$. After exposure to sunlight the values increased from S-2 to Set-4. The \% drug content in the Set-2, Set-3 and Set-4 was measured as 110.77, 112.50 and $140.40 \%$, respectively (Table-1, Figure-1). More importantly, the increase of $\%$ of chloramphenicol content of Set-2, Set-3 of product A compared to control (Set-1) was $5.08 \%$ and $8.36 \%$ respectively whereas for Set-4 the value increased up to $23.38 \%$. The UVabsorption spectrum of eye drops after exposure to sun exhibited a hypsochromic shift. The $\lambda \max$ of chloramphenicol before treatment (Standard) was 278 $\mathrm{nm}$ whereas the $\lambda$ max of sunlight-treated samples was found to be at $274 \mathrm{~nm}$. The absorption spectrum also showed a hyperchromic effect i.e. the absorption intensity also increases after sunlight degradation (Figure 3). 


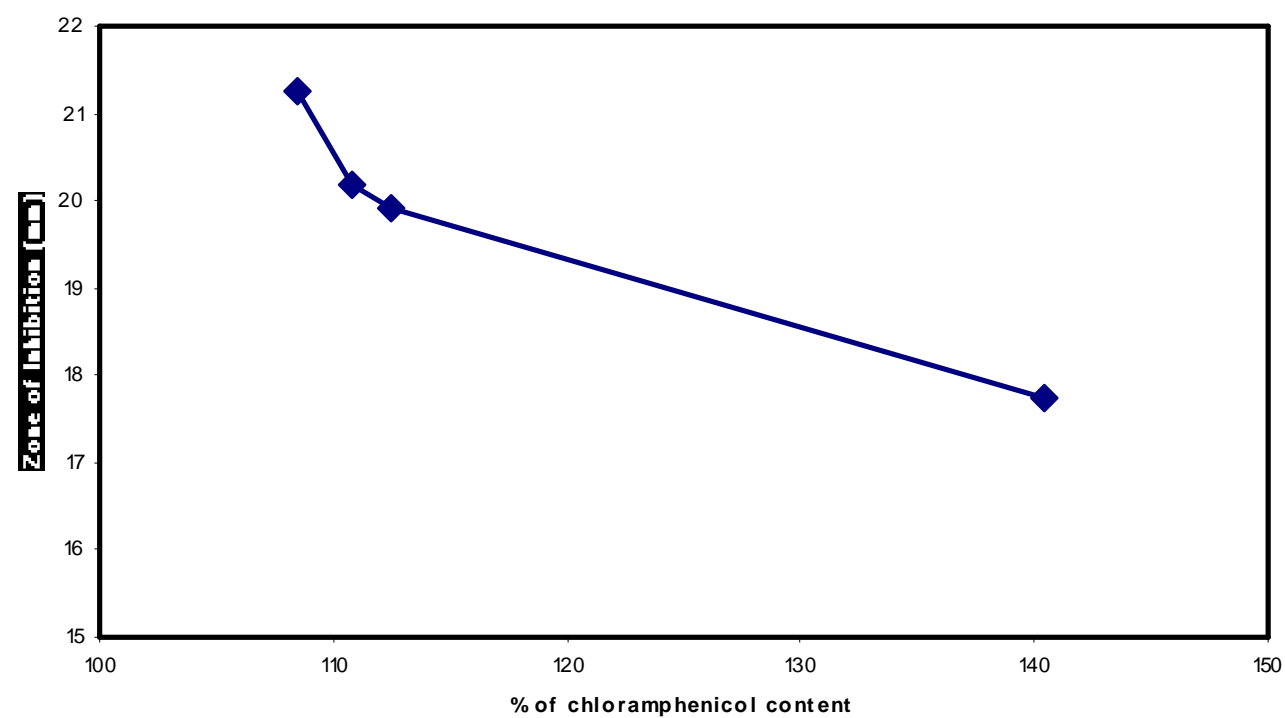

Figure 2. Relation between antibacterial activity against S. aureus and percent of chloramphenicol content in eye drops (product B) after exposure to sunlight.

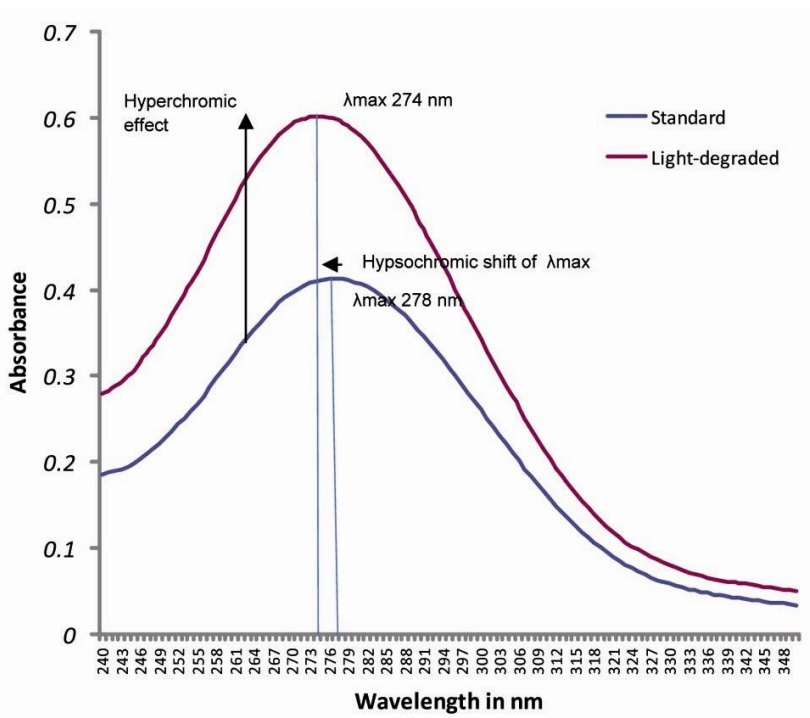

Figure 3. Shift of UV- absorption spectrum of chloramphenicol eye drop preparations before and after sunlight treatment.

The change in the zones of inhibition and percentage of decrease in efficacy of sunlight-induced authentic chloramphenicol solutions also showed the same trend as to the sunlight treated eye drop samples (data not shown). Aboshiha (2008) observed that as the chloramphenicol eye-drop not stored in refrigerator in the hot season of May, 2005 when the temperature was around $40^{\circ} \mathrm{C}$ became ineffective with presumed resistance of microbial infections (often in the absence of microbiological sensitivity data). The present microbiological study determining the loss of efficacy of the chloramphenicol eye drops strongly supports the previous findings.

\section{Conclusion}

An inverse correlation between the estimated drug content and the antibacterial activity was found which indicates that the chloramphenicol in the preparations exposed to sunlight are degraded and produced a colored compound responsible for higher absorbance in the specified wavelength $(278 \mathrm{~nm})$. The results suggest that in spite of being less expensive, pharmaceutical companies should not use the UV-spectroscopic method to analyze chloramphenicol. High Performance Liquid Chromatography (HPLC) as suggested by BP and USP should be followed for the analysis. Moreover, a costeffective and easy method like microbial assay method should be developed to assay the chloramphenicol content in the preparations which can be used by the middle-sized pharmaceutical industries who are not able to afford high cost equipments like HPLC. Thus, any chemical compounds that have degradation products having the UV absorption around the same $\lambda$-max of parent compound cannot be assayed by UV-spectrophotometric method. Microbiological assay must be performed to analyze the potency of antibacterial compounds like chloramphenicol. 


\section{References}

Aboshiha, J., Weir, R., Singh, P., Ewings, P. and Lovering, A. 2008. To what extent does a lack of refrigeration of generic chloramphenicol eye-drops used in India decrease their purity and what are the implications for Europe?, $\mathrm{Br} J$ Ophthalmol 92, 609-611.

Bauer, A.W., Kirby, W.M., Sherris, J.C. and Turck, M. 1996. Antibiotic susceptibility testing by a standardized single disk method. Am. J. Clin. Pathol. 45, 493-496.

Lawrence, D.R., Bennett, P. and Brown, M.J. 1987. Clinical Pharmacology, Churchill Livingstone,
Martindale, W. 1989. Martindale: The Extra Pharmacopoeia, Amer Pharmaceutical Assn.

Serrano, M.C., Ramírez, M., Morilla, D., Valverde, A., Chávez, M., Espinel-Ingroff, A., Claro, R., Fernández, A., Almeida, C. and Martín-Mazuelos, E. 2004. A comparative study of the disk diffusion method with the broth microdilution and Etest methods for voriconazole susceptibility testing of Aspergillus spp. J Antimicrob Chemother 53, 739-742.

Shih, IK. 1971. Degradation products of chloramphenicol, J. Phar. Sci. 60, 786-787.

Turk, DC. 1977. A comparison of chloramphenicol and ampicillin as bactericidal agents for Haemophilus injluenzae type-b. J. Med. Microbial. 10, 127-31. 